\title{
Design and Implementation of Portable NMR Probe Magnet
}

\author{
Gao Junxia*, Zhang Yiming, and Tian Jiashen \\ Faculty of Information Technology, Beijing University of Technology, Beijing 100124, China
}

(Received 28 December 2016, Received in final form 3 March 2017, Accepted 7 March 2017)

\begin{abstract}
The NMR's probe consists of the static magnetic field generator (magnetic source) and the RF coil. It is very strict for the homogeneity of the static magnetic field intensity of the magnetic source, so the cost of the magnetic source is more expensive in the entire nuclear magnetic resonance instrument. The magnetic source generally consists of electromagnet, permanent magnet and superconducting magnet. The permanent magnet basically needs not to spend on operation and maintenance and its cost of manufacture is much cheaper than the superconducting magnet. Therefore, the permanent magnet may be the only choice for the static magnetic field device if we want to use the magnetic resonance instrument as an analyzer for production by reducing price. A new probe magnet was developed on the basis of the permanent magnet ring in this paper to provide a technological way for reducing the manufacturing cost, weight and volume of the existing nuclear magnetic resonance instrument (including MRI) probe.
\end{abstract}

Keywords : permanent magnet, magnet ring, nuclear magnetic resonance, 2D field solution

\section{Introduction}

Since the nuclear magnetic resonance (NMR) was discovered by Bloch and Purcell IN 1946, NMR, as an important and modern analytical technique for studying matter properties, has got a quick application and development in physical chemistry, molecular biology, medical science, analytical chemistry and geology. The NMR instrument still cannot be used in production of industry and agriculture due to the cost of manufacture, but if the innovation and breakthrough of technology can significantly reduce the instrument price, the area or industry that the low field pulse NMR analyzer can be used in is as following: Agriculture and food industry, petroleum, chemical industry, textile industry, and medical diagnosis [1-4].

The hardware of the NMR instrument mainly consists of the probe and the electronic equipment. The probe consists of the static magnetic field generator (magnetic source) and the RF coil. It is very strict for the homogeneity of the static magnetic field intensity of the magnetic source, so the cost of the magnetic source is more expensive in the entire nuclear magnetic resonance instru-

(C)The Korean Magnetics Society. All rights reserved.

*Corresponding author: Tel: +8613521225393

Fax: +8601067396621, e-mail: gaojunxia@bjut.edu.cn ment. Moreover, the SNR of the NMR echo signal is approximately in direct proportion to the square of the static magnetic field intensity, so the static magnetic field's intensity shall not be too low in order to obtain a clear signal. In order to meet the requirements of high field intensity and homogeneity, the magnetic source generally consists of the electromagnet, permanent magnet and superconducting magnet. Only the permanent magnet basically needs not spend on operation and maintenance, and its cost of manufacture is much cheaper than that of the superconducting magnet. Therefore, the permanent magnet may be the only choice for the static magnetic field device if we want to use the magnetic resonance instrument as an analyzer for production by reducing price [5].

In this paper, the permanent magnet was used to design and develop a new probe magnet to provide a technological way for reducing the manufacturing cost, weight and volume of the existing nuclear magnetic resonance instrument (including MRI) probe. As a result, the system becomes smaller, easy to operate, low price and easier in popularization and application.

\section{Optimization Design of Probe Magnet}

The design of the probe magnet is based on improvement of the permanent magnetic ring. The permanent 
magnetic ring is a new permanent magnet structure (also called the Halbach array structure) suggested by J.C. Mallinson and K. Halbach, physicist of the Lawrence Berkeley National Laboratory [6, 7]. As the development demand for successive miniaturization and portability of the permanent magnetic device, the outstanding feature of the permanent magnetic ring has attracted broad attention of academic and industrial circles [8-12]. The absolute homogeneity of the internal magnetic field of the permanent magnetic ring lies in the basis that the permanent magnet material is continuously magnetized and has no external side leakage, so it is hard to reach by current manufacturing and processing technique of the permanent material. Therefore, it is often combined by many individually magnetized discrete magnets in the actual production. The permanent magnet has an individual difference when preparing, so the magnetic properties of the discrete magnets cannot be consistent ideally. Beyond that, under the effect of magnetic field force, magnets in some positions are hard to combine together, so it is inevitable that glue crevices will generate the leakage flux. The internal magnetic field of the actually combined permanent magnetic rings is not ideal [13]. This heterogeneous magnetic field will bring in a wide-band noise, which will result in reduction of instrumental sensitivity and even loss of the feedback signal when NMR has a high requirement for the accuracy of the static magnet source. Therefore, the main problem for current study on the permanent magnet ring is how to eliminate this homogeneity difference and enhance the available magnetic field space.

By analyzing the 2D field solution of the permanent magnetic ring, we can know that the two factors affecting the homogeneity of the internal magnetic field of the permanent magnetic ring are as follows: First, magnet assembly in some location is difficult to completely stitching together. There is the leakage flux caused by magnet gap; second, the magnetic properties of each magnet are not exactly the same and the magnetization direction is discontinuous. There are the magnetic field fluctuations. Therefore, it can improve the magnetic field homogeneity and enhance the utilization of the permanent magnet material by reducing the leakage flux or increasing the discrete frequency of the magnetization vector to inhibit the amplitude of the amplitude of low-order harmonics in the magnetic field fluctuations $[14,15]$. However, the frequently used permanent material like the neodymium iron boron is higher in the magnetic energy product and stronger in magnetic force, so the magnetic field force of the repulsive interaction between magnets that part of magnetization directions are similar is higher.
Even if the auxiliary fixing method is added, it will leave a space between discrete magnets in order to keep the permanent magnet a good geometrical shape.

Because the magnet gap and the leakage flux are inevitable, the main research contents for optimization of the permanent magnetic ring's magnetic field properties are changed to how to inhibit the low-order harmonics in the magnetic field fluctuations on the condition that the degree of filling of the permanent magnet is as high as possible. As for the permanent magnetic ring, the most direct way to inhibit the low-order harmonics is to add the number of magnet blocks, M. we can assume that the angle change of the magnetization vector between neighboring magnetic blocks is reduced, and it is the ideal magnetization when $\mathrm{M}$ is infinite. However, the simple increasing of quantity means the entire array's complexity will increase. In addition, the preparation of the permanent magnet material has an individual difference, so the higher the individual quantity is, the utilization of the permanent magnet material is lower in order to select the unit magnets with the magnetic properties as consistent as possible. The main research issue of this paper is how to optimize the geometrical shape and the magnetization direction to make it become closer to the magnetization of the ideal permanent magnetic ring when $\mathrm{M}$ is under a certain condition.

For the relationship between the $\mathrm{M}$ value and the magnetization vector, the representation can be simplified by Fig. 1(a).

The straight line through the origin represents the trend of the ideal magnetization vector in Fig. 1(a), i.e.:

$$
\beta(\varphi)=2 \varphi
$$

And the two polylines represent the change curves of the magnetization vector when $M$ is 8 and $M$ is 16 , respectively, in Fig. 1(a). When $M$ is 16, the information of the magnetization vector is richer and closer to the ideal straight line. In order to approximate this effect without increasing the $\mathrm{M}$ value, another 8 discrete polyline may be added on the polyline of $\mathrm{M}=8$, as shown in Fig. 1(b). Two polylines are spaced by a certain phase difference to compensate for a certain amount of magnetization vector information.

\subsection{Optimizing strategy of permanent magnetic ring}

In order to reflect the changes Fig. 1(b) into the actual permanent magnetic ring, according to the linear theory of the permanent magnet, we can cut a permanent magnetic ring composed of fan-shaped unit magnets that its external diameter is $r_{0}$, internal diameter is $r_{i}$, and $\mathrm{M}$ is 8 into $\mathrm{P}$ layer (s) along the radius (P may be any even). 


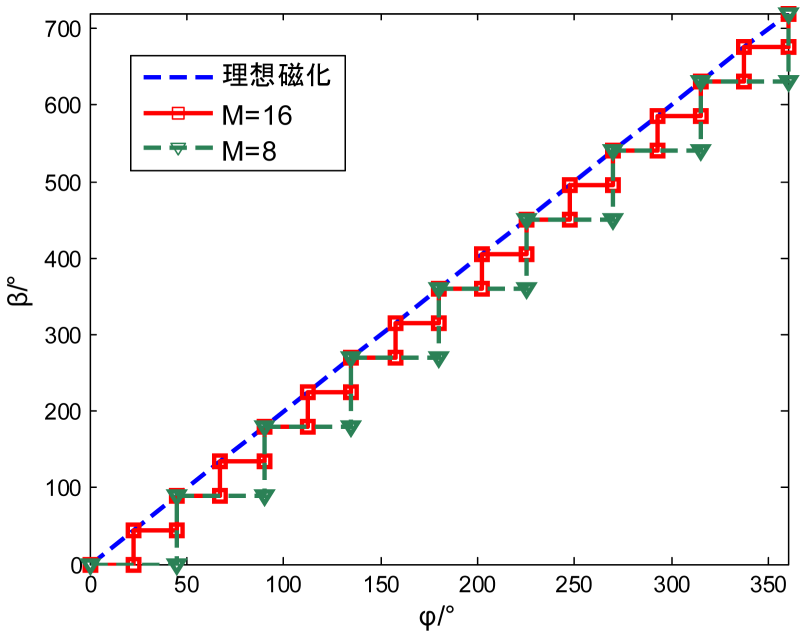

(a)

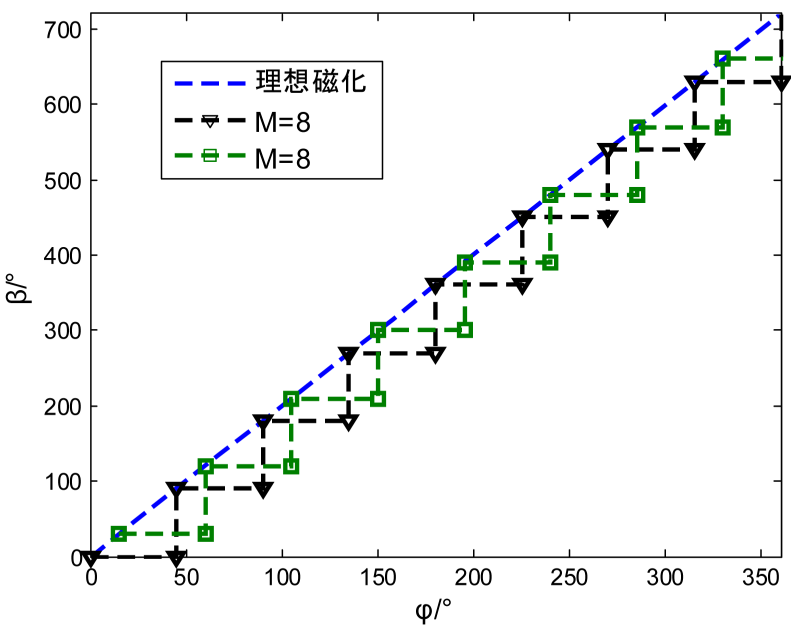

(b)

Fig. 1. (Color online) Relationship between the number of unit magnet and the magnetization vector.

Each layer we obtained is still a permanent magnetic ring that it is based on a fan-shaped unit magnet and has the same magnetization vector. As shown in Fig. 2(a), it is a layered structure that $P$ is 6 . Here we take $P=6$ as an example to illustrate. The layered structure is shown in Fig. 2(a).

According to the similarity principle of the permanent magnetic circuit, i.e., if two magnetic circuits are different in size but similar in geometrical shape, and the permanent magnet and the magnetizer are the same in property and their configurations are the same, the gap flux densities of the two magnetic circuits are equal. Therefore, for ease of future analysis, the rate of the internal diameter to the external diameter of each layer of the permanent magnetic ring is set to $d$, as following:

$$
d=\left(\frac{r_{i}}{r_{o}}\right)^{\frac{1}{P}}
$$

In order to compensate for the magnetization vector, we can clockwise turn each layer of the permanent magnetic ring to an angle outwards along the radius but the included angle of the magnetization vector to the coordinate axis is kept unchanged. As a result, a new structure, as shown in Fig. 1(b) can be obtained. The rotation angle of the neighboring layers is the same. Where the angle of the outermost layer is $\alpha$, compared to the innermost layer.

This new structure is based on the change of the fanshaped unit magnets. To ensure that the largest possible permanent magnet material filling, and to some extent to do the redistribution of magnetization vector, so that the magnetization vector is more homogeneous in the permanent magnetic ring, under the same number of discrete and units magnet. The change in the angle of each layer of permanent magnetic ring will cause changes in the phase of the each harmonic in the magnetic field fluctuations. These changes in phase are beneficial for suppressing the amplitude of the first harmonic. From Fig. 2(b), it can be found that the distribution of the magnetization vector in the permanent magnet is related to the number of layers $\mathrm{P}$ and the rotation angle $\alpha$ of the whole permanent magnetic ring.

In Fig. 2(b), if a group of magnets having the same magnetization direction in the radial layered structure are taken out, as shown in Fig. 3. Wherein the rotation angle of the $j$-th layer structure relative to the innermost first layer is $\theta$ and the rate of the internal diameter to the external diameter is same. But the rotation angle of the $j+1$-th layer structure relative to the $j$-th layer is $d \theta$. Since the direction of the magnetic induction strength in the pre-optimized magnet is in the positive direction along the $\mathrm{Y}$ axis and the rate of change of the magnetization direction of the single-pole permanent magnetic ring is

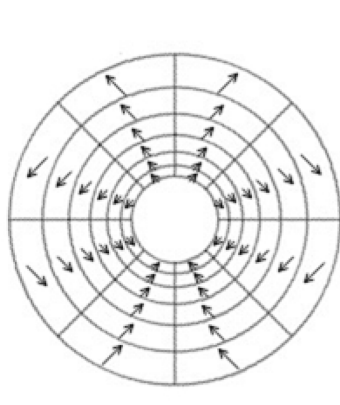

(a)

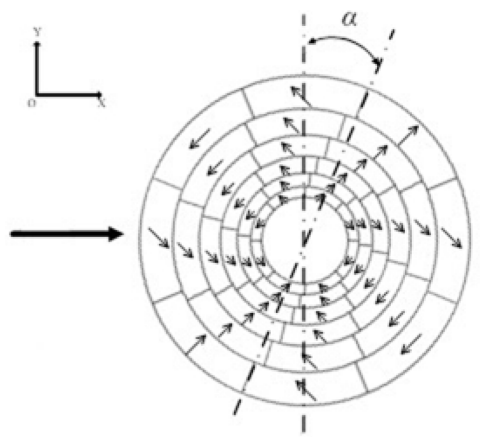

(b)
Fig. 2. Distribution mode of optimized magnetization vector. 


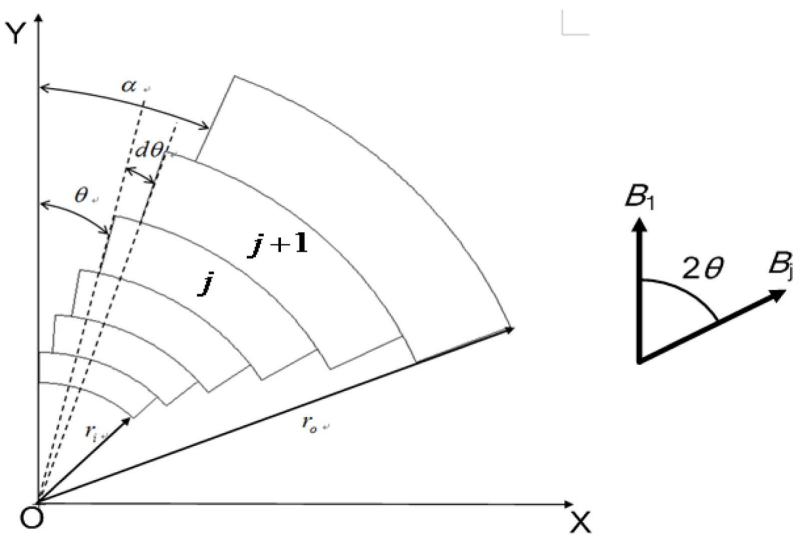

Fig. 3. A set of magnets with the same magnetization vector.

twice the rate of change of the magnet angle, the amount of change in the magnetization direction of the $j$-th layer of permanent magnetic ring is $2 \theta$. According to the easy rotation axis theorem, the magnetic induction strength $B_{j}$ produced by the $j$-th layer at the origin is compared with the magnetic induction strength $B_{1}$ produced by the first layer in permanent magnetic ring, and the change of angle is $-2 \theta$, and the magnitude is constant.

By comparing simulation of different $\mathrm{P}$ values, the five relationships between angle $\alpha$ and amplitude of the first harmonic are obtained, as shown in Fig. 4.

It can be seen that with the increase of angle $\alpha$, the amplitude of the first harmonic decreases and the inhibitory effect is enhanced. The distribution of magnetic induction strength of the magnetic field in permanent magnetic ring is more ideal. With the increasing of $\mathrm{P}$ values, the simulation results tend to a fixed curve. When $\mathrm{P}$ is large enough, the geometries of the unit magnets having the same magnetization in Fig. 3 will become the crescent

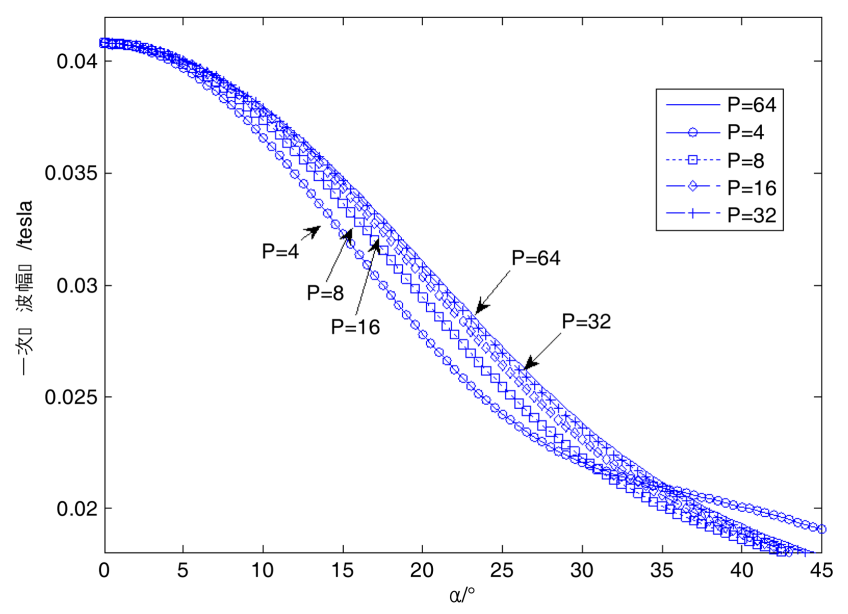

Fig. 4. (Color online) Relationship between angle $\alpha$ and amplitude of the first harmonic with different $\mathrm{P}$.

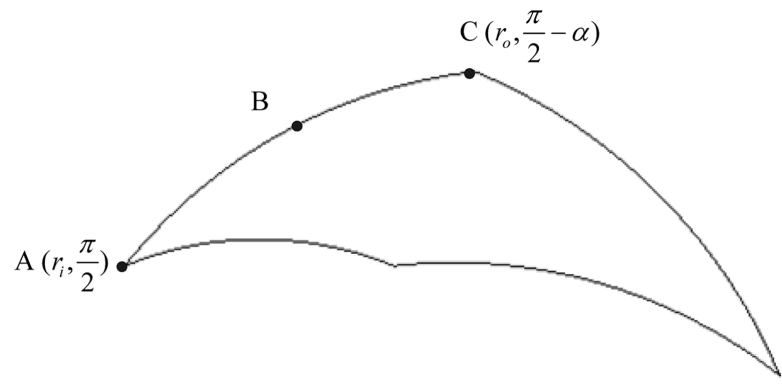

Fig. 5. The crescent magnet in polar coordinates.

shapes in Fig. 5. Corresponding to the previous singlepole permanent magnet ring composed of fan-shaped unit magnets, this new structure is referred to as crescentshaped permanent magnetic ring in later discussion.

When processing permanent magnetic rings, the unit magnets are generally obtained in such a way that the permanent magnet blank is cut by a wire cutting machine and magnetized by a magnetizer. The currently wide-used sintered Nd-Fe-B permanent magnet is an anisotropic material, i.e., as for the prepared workblank, the internal magnetization direction is already definite. If external magnetic fields of other directions are added during magnetizing, it may cause a severe leakage flux. Therefore, the unit magnets of the permanent magnetic ring shall be cut according to the determined shape and magnetization direction followed by magnetizing and bonding. Whether a unit magnet is fan-shaped or crescent-shaped, and if it is cut from a standard cubic blank, it is inevitable that a lot of waste will be produced. In order to improve the utilization of the permanent magnet material and reduce the processing cost, a unit magnet structure of the isosceles triangle is suggested in this paper [16]. It can be regarded as a special form of the crescent-shaped unit magnet at an angle $\alpha$ of $45^{\circ}$, and its cross-section is as shown in Fig. 6.

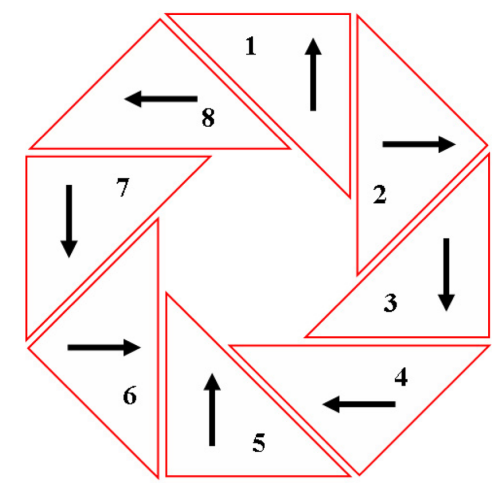

Fig. 6. (Color online) Structure of triangular magnet. 


\section{Finite Element Simulation Comparison}

In order to compare the performance differences of the permanent magnet ring composed by isosceles right triangle, fan-shaped, and crescent-shaped structure, this article used the Maxwell of Ansoft, a company of finite element simulation software, to build the models for simulation [16-23]. The magnet parameter referred to the previous setting that the minimum inner diameter was 25 $\mathrm{mm}$ and the minimum outer diameter was $40 \mathrm{~mm}$. The selected material was neodymium iron boron (N35). The distribution of magnetic flux density of the fan-shaped, crescent-shaped, and isosceles right triangle structures in the air is shown in Figs. 7 9(a), and the distribution of

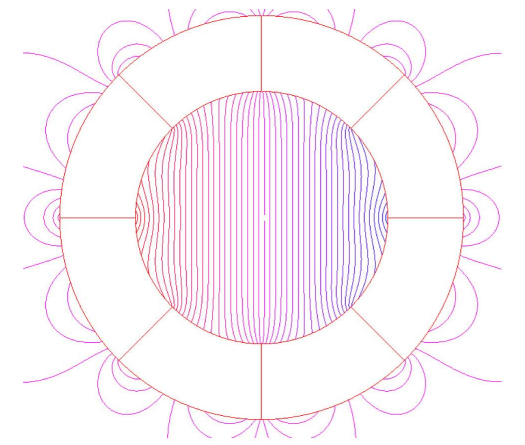

(a)

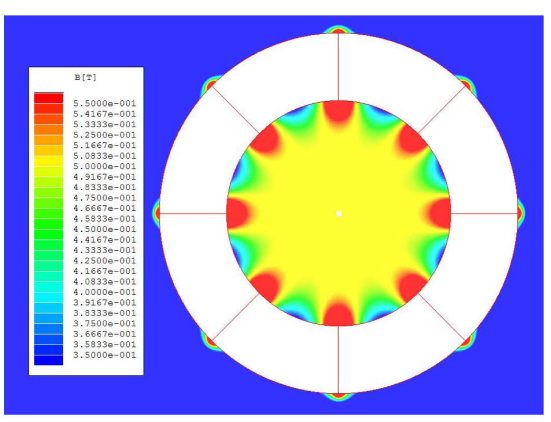

(b)

Fig. 7. (Color online) Distribution of magnetic flux density and magnetic induction strength of the fan-shaped structure.

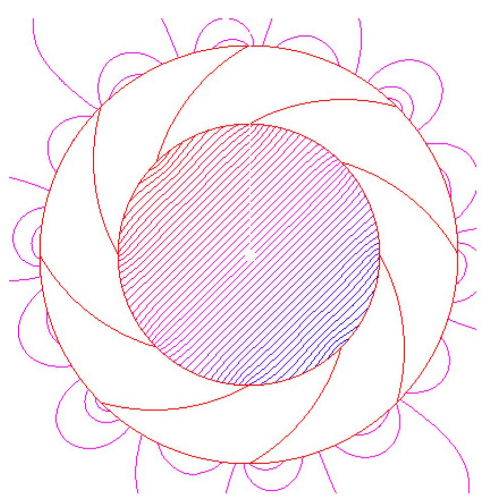

(a)

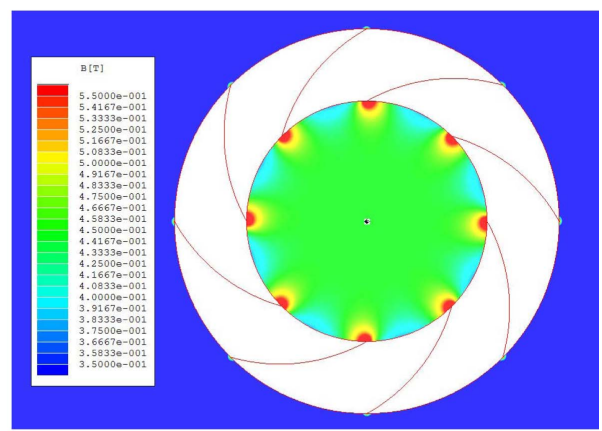

(b)

Fig. 8. (Color online) Distribution of magnetic flux density and magnetic induction strength of the crescent-shaped structure.

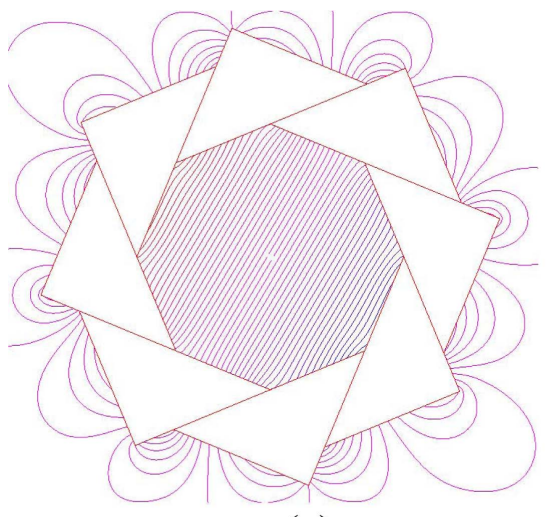

(a)

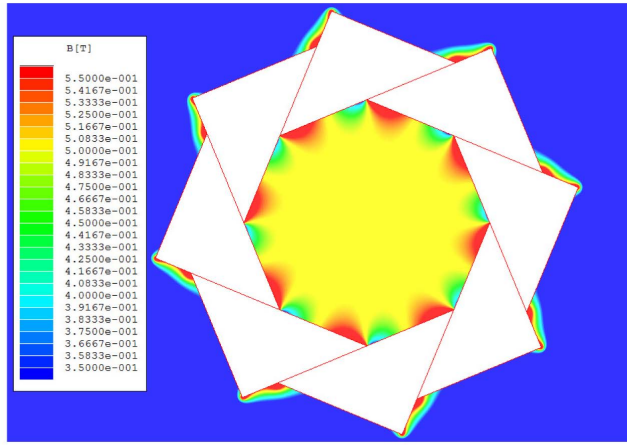

(b)

Fig. 9. (Color online) The distribution of magnetic flux density and magnetic induction strength of the isosceles right triangle structure (The color bar is the same scale in Fig. 7-9). 


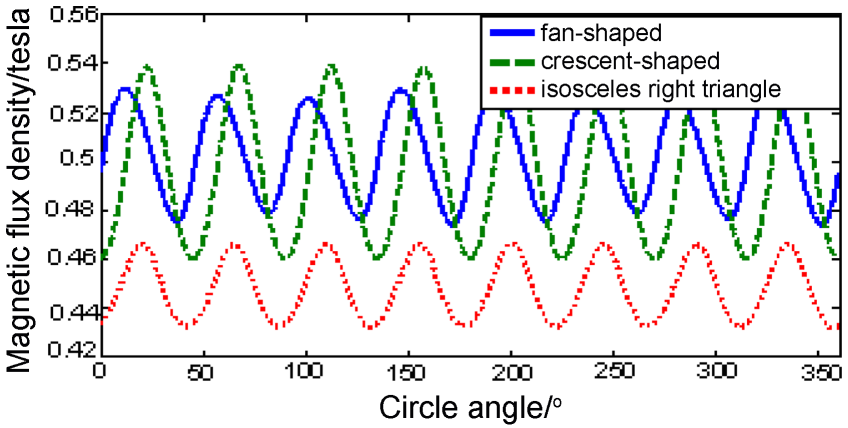

Fig. 10. (Color online) Distribution of the magnetic induction strength of $\mathrm{r}=20 \mathrm{~mm}$ circumference of three different structures.

magnetic induction strength is shown in Figs. 7 9(b).

Seeing from the results, the magnetic flux density and the $\mathrm{Y}$ axis forward angle of the crescent-shaped and isosceles right triangle structure increased when compared to the fan-shaped structure; the currents were smoother. In the magnetic induction strength distribution cloud diagram, the inner magnetic induction strength of the adjacent discrete magnet bounding area decreased and the protruding dark semicircle area reduced. Although the magnetic induction strength nears the origin point, the magnetic field area with propinquity color of the central area increased. That meant the evenness of the magnetic field increased. In addition, the magnetic flux leakage of the outside of the discrete magnet bounding area was better improved than the permanent magnet ring before optimization.

The distribution of the magnetic induction strength of $r=20 \mathrm{~mm}$ circumference and the infinite element simulation results of the fan-shaped and crescent-shaped permanent magnet ring $\left(\alpha=45^{\circ}\right)$ is shown in Fig. 10.

From Fig. 10, under the unchanged condition of the inner diameter of permanent magnet ring, the average thickness of the permanent magnetic ring composed of the isosceles right triangle unit magnets is increased with the thickness of the permanent magnetic ring composed

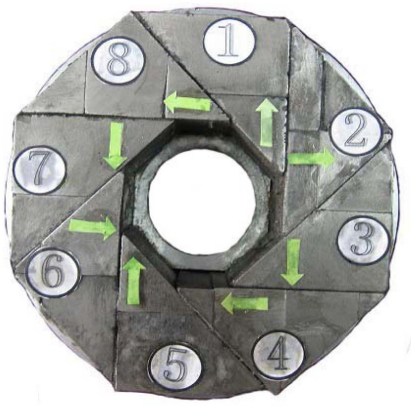

Fig. 11. (Color online) Probe magnet of 8 cross-section isosceles right triangles.

of the fan-shaped and crescent-shaped unit magnets. Therefore, in terms of the magnet induction strength on the same radius circumference, the actual DC component would increase comparing to the crescent-shaped structure. Basically it was quite the same as the fan-shaped structure before improvement; the inhibition effect of the first harmonic $\left(n^{\prime}=8\right)$ is worse than crescent-shaped structure.

In addition, in order to observe the degree of difficulty when assembling the permanent magnet ring structure, with the help of finite element simulation software, the magnetic field force and polar coordinate angle received in the matrix of any unit magnet is shown in Table 1. Refer to Fig. 6 for the magnet numbering.

Therefore, whether it was the evenness of the magnetic field or the magnetic field force, the isosceles right triangle permanent magnet ring could be as a compromised solution. Considering greatly reducing the process difficulty and manufacturing cost, this structure was nonetheless a better choice.

\section{The Manufacture of Probe Magnet and Performance Test}

A probe magnet was manufactured according to the magnetizing direction shown in Fig. 6, i.e. the improved

Table 1. Force received by the unit magnet of permanent magnet ring of different structures.

\begin{tabular}{|c|c|c|c|c|c|c|}
\hline \multirow{2}{*}{$\begin{array}{l}\text { Magnet } \\
\text { number }\end{array}$} & \multicolumn{2}{|c|}{ Fan-shaped } & \multicolumn{2}{|c|}{ Isosceles right triangle } & \multicolumn{2}{|c|}{ Crescent-shaped $\left(\alpha=45^{\circ}\right)$} \\
\hline & Magnet force $(\mathrm{N})$ & Angle $\left(^{\circ}\right)$ & Magnet force $(\mathrm{N})$ & Angle $\left({ }^{\circ}\right)$ & Magnet force $(\mathrm{N})$ & Angle $\left(^{\circ}\right)$ \\
\hline 1 & 6033.24 & 204.69 & 3743.28 & 199.75 & 2434.39 & 158.88 \\
\hline 2 & 6030.83 & 245.42 & 3709.51 & 243.88 & 2295.34 & 201.21 \\
\hline 3 & 5649.12 & 290.67 & 3596.23 & 289.62 & 2180.30 & 249.63 \\
\hline 4 & 5626.48 & 340.01 & 3701.28 & 339.01 & 2331.97 & 297.52 \\
\hline 5 & 6009.48 & 24.96 & 3862.83 & 20.28 & 2435.92 & 338.90 \\
\hline 6 & 6046.16 & 65.16 & 3710.92 & 63.83 & 2294.51 & 21.17 \\
\hline 7 & 5632.21 & 110.03 & 3596.06 & 109.87 & 2183.74 & 69.67 \\
\hline 8 & 5644.87 & 157.54 & 3703.27 & 158.98 & 2330.48 & 117.57 \\
\hline
\end{tabular}




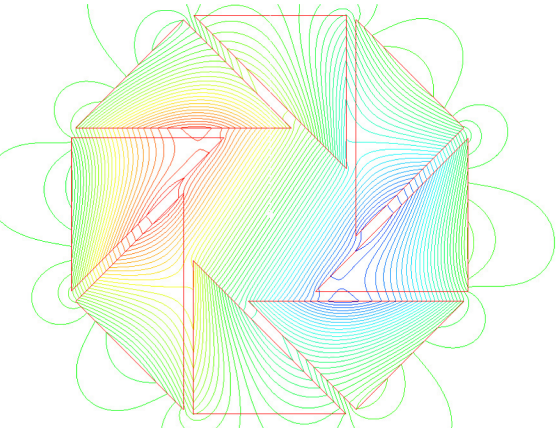

(a)

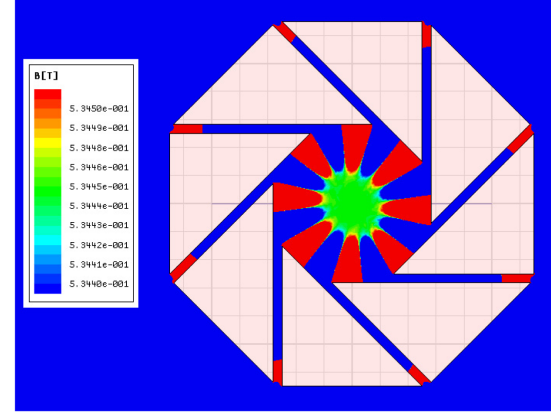

(b)

Fig. 12. (Color online) The distribution of magnetic flux density and magnetic induction strength of the isosceles right triangle structure.

permanent magnet ring, as shown in Fig. 11. The magnet was composed of 8 cross-sections of isosceles right triangles, each of which had $50 \mathrm{~mm}$ isosceles length and $70.7 \mathrm{~mm}$ base length, center inner diameter was $28 \mathrm{~mm}$, outer diameter was $65 \mathrm{~mm}$, magnet gap was approximately $1 \mathrm{~mm}$. The material was neodymium iron boron (N35). The magnetizing direction of each magnet block is shown in the direction of the arrow in the Fig. 11.

In order to verify the performance of the probe magnet, we adopted the commercial electric magnetic 2D field computing software, Maxwell 2D, provided by Ansoft Company. The residue magnet and the coercivity was set at the average value of the 11 magnet blocks residue magnet, which were $0.94 \mathrm{~T}$ and $8.24 \mathrm{kOe}$. The adhesive gap of the adjacent magnet blocks was $1 \mathrm{~mm}$ and the corecivity amount was 1.05 . The distribution of the magnetic flux density and magnetic field strength of the cylinder magnet composed of isosceles right triangle are shown as Fig. 12(a) and (b).

The range satisfying 100PPM in Fig. 12(b): 0.534398T

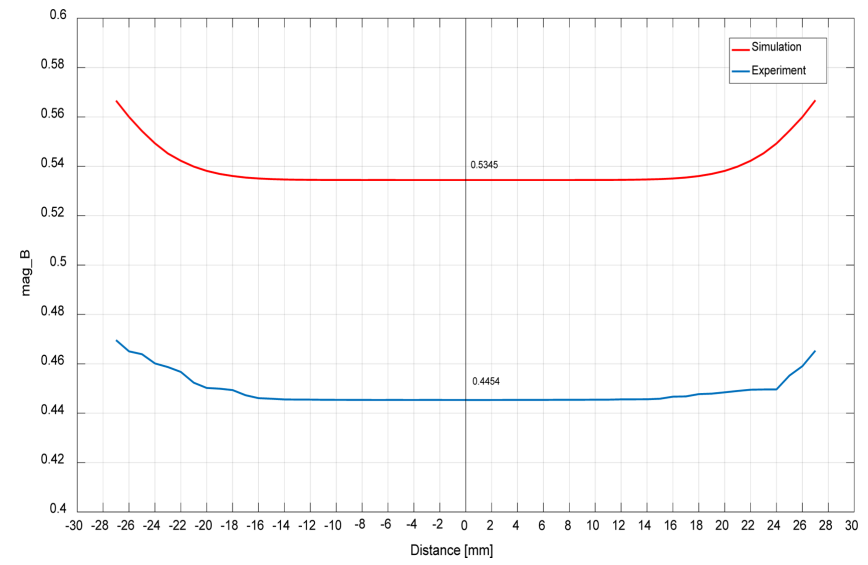

Fig. 13. (Color online) Simulation results and measurement results.
$0.534504 \mathrm{~T}$, the radius of the available space was $1.2 \mathrm{~cm}$. The distribution of the magnetic induction strength along the radial straight line starting from the center is shown in the red curve in Fig. 13.

Then, test the probe magnet. The block diagram of test system is shown in Fig. 14. The composition diagram of test system is shown in Fig. 15. Use Gausses Meter and the Mechanical and Electrical Engineering Department, Beijing University of Technology self-developed coordinate measuring instrument to test the magnet induction strength in the cylinder. Secure the probe of the Gauss Meter on the suspending arms of the coordinate measuring instrument vertically downward and then place the magnet standard. Position with the coordinate measuring instrument and mark the axial $25 \mathrm{~mm}$. Measure the magnetic induction strength in the $0.1 \mathrm{~mm}$ interval from the center along the radius. The measurement results are shown in the blue curve in Fig. 13.

Fig. 13 shows that the measurement results and the simulation results of the probe magnet have $16.65 \%$ differences. The main reasons are that the magnets that make up the permanent magnetic ring have certain individual differences in the preparation, and the magnetic properties of the magnets cannot achieve the ideal consistency. Beyond that, under the effect of magnetic field force,

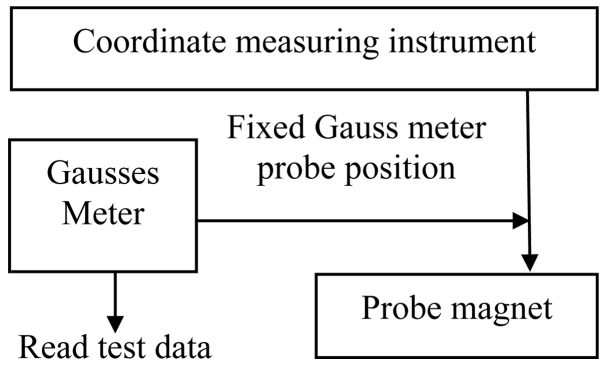

Fig. 14. (Color online) The block diagram of test system. 


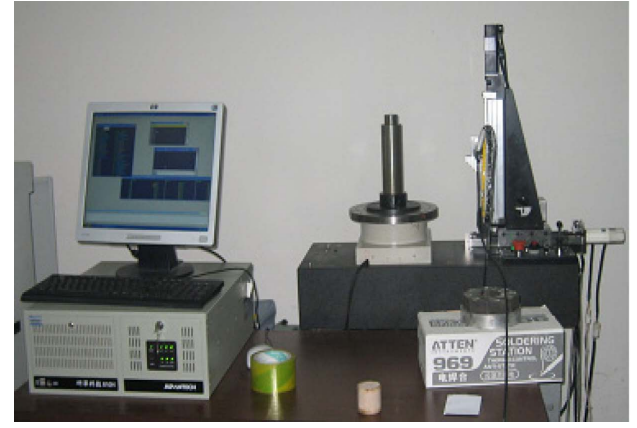

Fig. 15. (Color online) The composition diagram of test system.

magnets in some positions are hard to combine together, so there is leakage flux between glue crevices, and the surrounding magnetic field environment.

\section{Conclusions}

This article proposed a new probe magnet of portable nuclear magnetic resonance instrument structure by improving the permanent magnet ring. Maxwell 2D of infinite element simulation software company, Ansoft, was used for simulation. The simulation results showed that the permanent magnet ring of fan-shaped could effectively increase the evenness of the inner magnetic field when using the improved magnetic structure. Comparing to the crescent-shaped permanent magnet ring, the improved magnetic structure increased the using rate of the permanent magnet material and was easy to process. Used neodymium iron boron to manufacture a probe magnet and compared the simulation results and the measurement results. The main reasons of differences were that the magnets that make up the permanent magnetic ring have certain individual differences in the preparation, and the magnetic properties of the magnets cannot achieve the ideal consistency. Beyond that, under the effect of magnetic field force, magnets in some positions are hard to combine together, so there is leakage flux between glue crevices, and the surrounding magnetic field environment.

\section{Acknowledgement}

This work was supported by R\&D of portable NMR probe technology (the National Ministries R\&D Projects), Grant No. 20121103110020.

\section{References}

[1] Q. S. Xiao and J. Y. Zhu, Petroleum Geology \& Experiment. 31, 97 (2009).

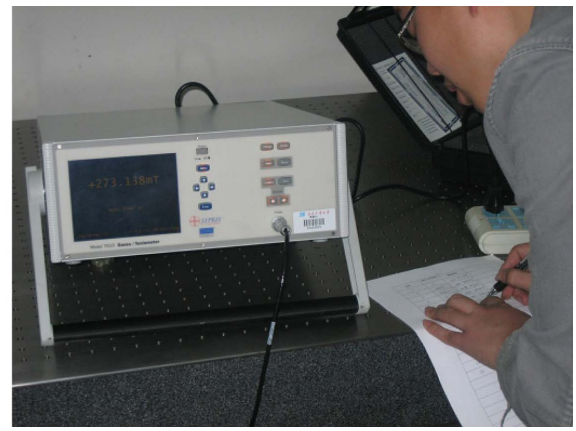

[2] Z. R. Ni, X. H. Cui, S. G. Sun, and Z. Chen, Spectroscopy and Spectral Analysis 31, 1 (2011).

[3] J. Lin, Q. M. Duan, and Y. J. Wang, Theory and Design of Magnetic Resonance Sounding Instrument for Groundwater Detection and ITS Applications, 1st Ed., Beijing Science Press, Beijing (2011).

[4] Y. M. Zhang, P. C. Xia, and Y. J. Yu, IEEE Trans. Appl. Supercond. 10, 763 (2000).

[5] H. B. Gao and Z. F. Zhang, Principle and Experimental Method of Nuclear Magnetic Resonance, 1st Ed., Wuhan University Press, Wuhan (2008).

[6] J. C. Mallinson, IEEE Trans. Magn. 9, 678 (1973).

[7] K. Halbach, Nuclear Instruments and Methods 169, 1 (1980).

[8] C. S. Li, W. Wang, and Y. M. Du, J. Eng. Design. 15, 33 (2008).

[9] H. Jing, J. Wang, S. Wang, L. Wang, and L. Liu, Physical C: Superconductivity and Its Applications 426 (2007).

[10] R. Bjørk, A. Smith, and C. R. H. Bahl, J. Magn. Magn. Mater. 384, 128 (2015).

[11] C. K. Chandrana, J. A. Neal, D. Platts, B. Morgan, and P. Nath, J. Magn. Magn. Mater. 381, 396 (2015).

[12] S. J. Lee, J. M. Kenkel, and D. C. Jiles, IEEE Trans. Magn. 38, 2991 (2002).

[13] J. Z. Chen and C. Y. Xu, IEEE Trans. Magn. 43, 3555 (2007).

[14] R. Bjork, C. R. H. Bahl, A. Smith, and N. Pryds, J. Appl. Phys. 104, 1 (2008).

[15] F. Bloch, O. Cugat, and G. Meunier, IEEE Trans. Magn. 34, 2465 (1998).

[16] J. Z. Chen, Y. M. Zhang, and C. Y. Xu, the Eighth International Conference on Electronic Measurement and Instruments (2007) pp. 330-336.

[17] D. Z. Qiao, Research and Optimization of Halbach Array based on Equivalent Magnetic Circuit Network Method. Ph.D. Thesis, Beijing University of Technology, Beijing (2010).

[18] X. Wang and Z. P. Wang, Power System Protection and Control. 37, 11 (2009).

[19] H. M. Liang, J. X. You, X. R. Ye, and G. F. Zhai, Trans. of China Electrotechnical Society 26, 46 (2011). 
[20] L. J. Chi, Y. Yan, and Z. W. Wen, Power System Protection and Control. 39, 151 (2011).

[21] W. Y. Yang, W. B. Ren, G. F. Zhai, and Q. X. Li, Trans. of China Electrotechnical Society 26, 51 (2011).

[22] Y. J. Song, M. Zhang, and Y. Zhu, Trans. of China Elec- trotechnical Society 29, 11 (2014).

[23] G. C. Zhang, C. Y. Liu, Q. Y. Chen, F. Chen, and X. D. Yang, Journal of South China University of Technology 42, 3 (2014). 